\title{
Evaluation of Quality control systems for X-Ray machines at different Hospitals using patient's radiological dose assessment technology
}

\author{
RadyAzzoz, Khaled M. ElShahat, Rezk Abdel MonemRezk \\ Must University - faculty of Applied Science-Radiation department \\ Faculty of Medicine - Al Azhar University-Radiation Oncology Department. \\ Physics Department- Faculty of Science - Helwan University
}

\begin{abstract}
The quality control tests' methods, as well as the criteria for scoring the results, are in full agreement with those specified in the American Association of Physicists in Medicine (AAPM) Report No.4 and IEC 61223-3-1 (AAPM, 1981; IEC 61223-3-1, 1999). There are a number of recent studies about dose optimization. Some of them are the surveys about image quality and patient dose in radiographic examinations in the authors' countries (Bouzarjomehri, 2004; Ciraj et al., 2005; Ramanandraibe, 2009; Papadimitriou, 2001; Shahbazi-Gahrouei, 2006). Some investigators focused only patient dose optimization (Brix et al., 2005; Vano\& Fernandez, 2007; Seibert, 2004; Williams \&Catling, 1998), whereas the others examined both the patient dose and image quality in radiographic devices (Aldrich et al., 2006; Schaefer-Prokop et al., 2008; Geijer, 2002). There are also studies that give reference values for clinical $x$-ray examinations by measuring phantom dose (Gray et al., 2005). But there is no any study focused to the dose optimization during quality control tests of $x$-ray devices. Dose optimization is very important because of the quality and quantity of quality control tests of x-ray equipmentconclusion this study shows that optimization of technical factors may lead to a substantial dose reduction. If the optimized parameters are applied to X-ray equipment during quality control tests, it is possible to determine how much good image quality will be obtained with this optimized parameters and how much dose will be measured when this qualified image is developed. The results show the importance of radiographic staff training about the recommended parameters that are applied to the x-ray units for a qualified quality control system. It is essential to provide relevant education and training to staff in the radiology departments. It can be sure that with such a study the questions on many professional staff's mind will be answered, and the dose and the image characteristics will be parameters that are controlled and managed.
\end{abstract}

Key Words: Quality Control - X-ray-Dose Measured-Optimization

\section{Introduction}

Radiation is a major risk in diagnostic and therapeutic medical imaging. The problem is caused from incorrect use of radiography equipment and from the radiation exposure to patients much more than required. Exposure of different dose values for the same clinical examination is an enough reason to draw attention to this issue. International Commission on Radiation Protection (ICRP), the International Atomic Energy Agency (IAEA) and other various independent institutions have been making publications in relation to ionizing radiation protection for more than fifty years. Report 60 of the ICRP and the Basic Safety Standards that was published in the IAEA report have three basic principles related to the radiation protection (ICRP, 1991; IAEA, 1996).

\section{Material and Methods}

Dosimax Plus A (Wellhöfer, Scanditronix, IBA, Germany) dosimeter was used to measure radiation dose.Dosimax Plus A dosimeter is a universal basic device and is designed according to IEC 61674 for acceptance tests and for quality checks at radiographic X-ray units. In Dosimax Plus A, dose measurements are performed by using solid state detectors (RQA). The dose range is from 200nGy to $9999 \mathrm{mGy}$ (IbaDosimetry, 2008). 


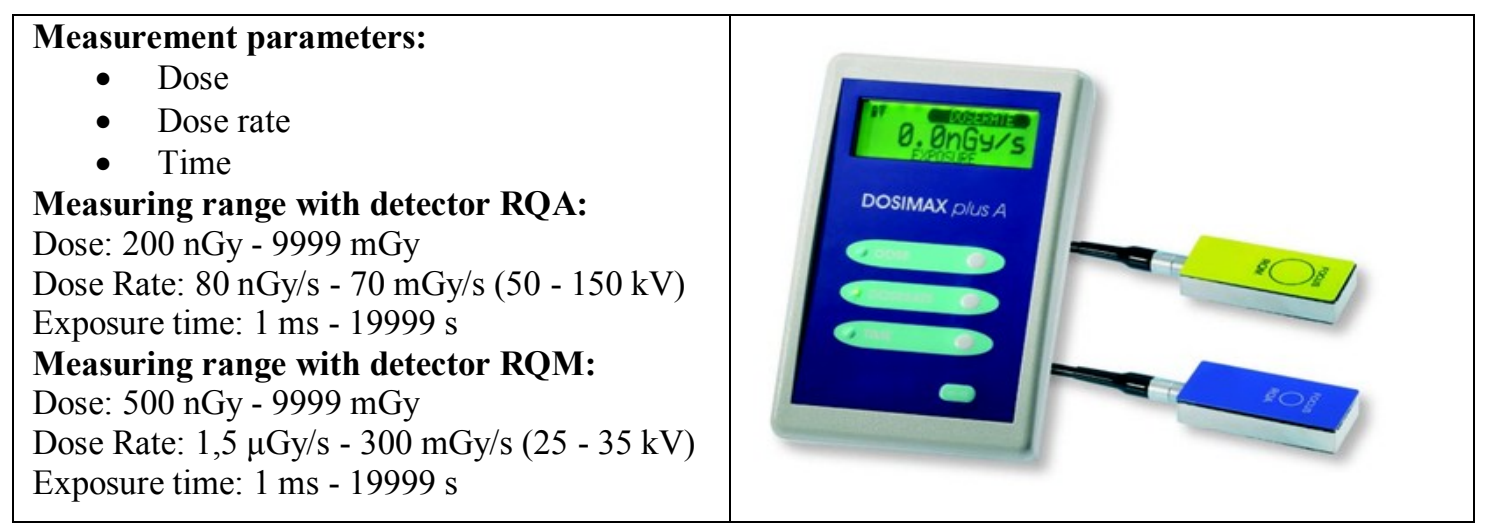

The radiographic measurements were performed in ten stationary X-ray units in eight hospitals. The Xray units including: Siemens, Philips, Toshiba, General Electric and Shimadzu were participated in this study. The reason for choosing these x-ray units is that their age is between 5 and 7 years old and the machines have 3 phase generators, thus their HVL value is kept in a narrow range, such as between 3 and 3,2mmAl.

It was calibrated by the Iba Laboratory of Germany and found to be capable of performing within recommended level of precision and accuracy

1- Beam Alignment and Collimator Accuracy for radio-diagnosis machine

2- Constancy of radiation output and linearity of $\mathrm{mR} / \mathrm{mAs}$ versus $\mathrm{KV}^{2}$ (small and large focus)

1. Assessment of total beam filtration

2. Assessment of focal spot size

3. Accuracy and constancy of exposure timer

4. Measuring of scattered radiation (using water phantom)

5. Leakage radiation from $x$-ray tube

\section{1-Constancy of Radiation output and linearity of $\mathrm{mR} / \mathrm{mAs}$ versus $\mathrm{kV}$ (Smal and large focus )}

Dose measurement applications have been included in recent recommendations (AAPM,1981; IEC 61223-3-1, 1999). The measurement procedures that wererealized in this study, areexplained below step by step.Before starting dose measurements, $\mathrm{kVp}$ accuracy tests were performed for 10 units and itwas seen that they have acceptable accuracy in according to the standards (AAPM, 2002).

Table (1 ) : Output for different KVp for one machine 1

\begin{tabular}{cccccc}
\hline kV Selected & kVpAvg.S.F & kVp Avg. L.F & kVp (Avg)2 & Output & Output (mR)/mAs \\
\hline 40 & 41.78 & 45.87 & 2.10 & 22.6 & 1.13 \\
60 & 66.8 & 65.35 & 4.27 & 56.4 & 2.82 \\
80 & 92.28 & 89.65 & 8.04 & 106.5 & 5.325 \\
100 & 109.4 & 110.2 & 12.14 & 205.1 & 10.255 \\
120 & 136.6 & 129.9 & 16.87 & 265.4 & 13.27 \\
\hline
\end{tabular}

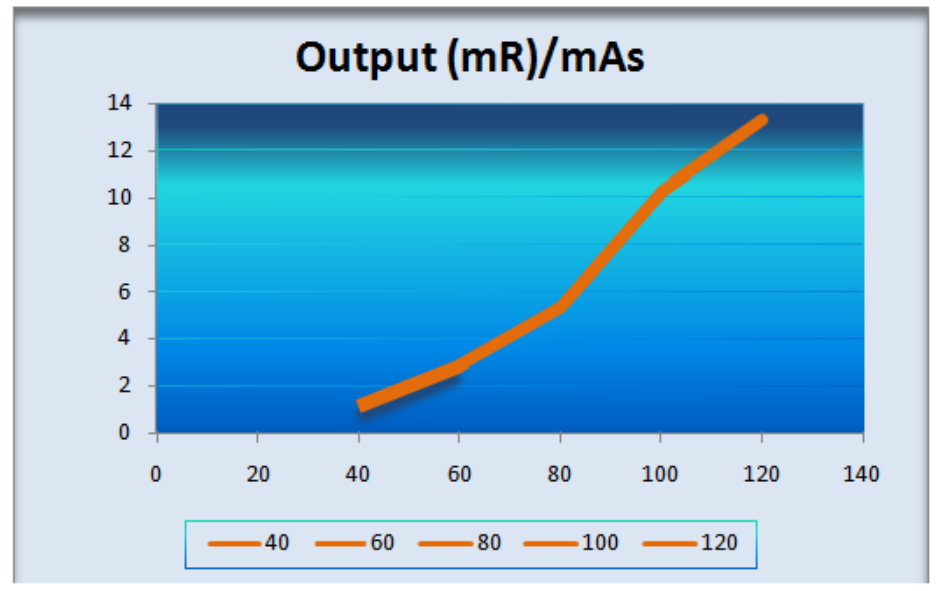

Fig (1) :Output for different KVp for one machine 1 
2-Constancy of Radiation output and linearity of $\mathrm{mR} / \mathrm{mAs}$ versus $\mathrm{kV}$

- The $\mathrm{mR} / \mathrm{mAs}$ relationship must be linear and acceptable

- $\quad \mathrm{kVp}$ measured by the $\mathrm{kV}$ Meter must be within the acceptable range (i.e. $+10 \%$ of $\mathrm{kVp}$ indicated by machine)

- $\quad$ The exposure dose (Output) must be within acceptable limits.

3. Assessment of Total Beam Filtration / (HVL)

HVL(Half Value Layer): is the most frequently used factor for describing both the penetrating ability and the penetration through specific objects. Defined: thickness of material penetrated by one half of the radiation

Unit: $\mathrm{mm}$ or $\mathrm{cm}$

Filtration: attenuation of photon according to their Energy ( simply the ability to get rid of unnecessary radiation

\begin{tabular}{|c|c|c|}
\hline AL mm & Output (+ 2) & $\mathbf{X i} / \mathbf{X 2}$ \\
\hline \hline $\mathbf{0}$ & 112.1 & 1.00 \\
\hline $\mathbf{1}$ & 83 & 0.74 \\
\hline $\mathbf{2}$ & 65.2 & 0.58 \\
\hline $\mathbf{3}$ & 57.1 & 0.51 \\
\hline $\mathbf{4}$ & 49.1 & 0.44 \\
\hline
\end{tabular}

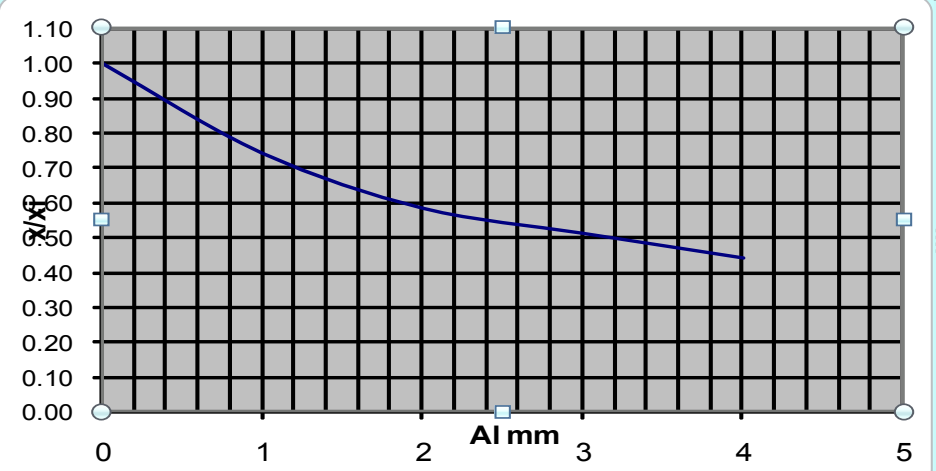

Fig ( 2 ): Total Beam Filtration AL( mm)

Entrance Surface Dose in different Hospital (mR)

-Assessment of X-ray dose variation with $\mathbf{k V p}$ the measured doses by changing $\mathrm{kVp}$ are given in Table 1. During measurements, mAswas firstly kept stable (20mAs) and $\mathrm{kVp}$ was changed as 50, 70, 80 and $100 \mathrm{kVp}$ to investigate the effects of $\mathrm{kVp}$ to the dose at stable mAs. After this, the same measurement procedure was applied to other mAs values (40 and 50mAs). All measurements were performed at distance of 100 and $60 \mathrm{~cm}$. Graphical representations of the relationship between dose and $\mathrm{kVp}$ value for constant $\mathrm{mAs}(40$ and $60 \mathrm{mAs})$ at $100 \mathrm{~cm}$ and $60 \mathrm{~cm}$ are given in Fig. 1, 2, and Fig. 3, 4, 5 , respectively.

$-$ different KVp

Table (3 ) : Measured Dose for different 8 X -ray Machines with value of $20 \mathrm{mAs}$ with

\begin{tabular}{|c|c|c|c|c|c|c|}
\hline Unit & $60 \mathrm{KVp}$ & $70 \mathrm{KVp}$ & $80 \mathrm{KVp}$ & $90 \mathrm{KVp}$ & $100 \mathrm{KVp}$ & $120 \mathrm{Kvp}$ \\
\hline 1 & 736 & 1345 & 2011 & 1990 & 5466 & 9241 \\
\hline 2 & 720 & 1356 & 2340 & 2345 & 4560 & 9451 \\
\hline 3 & 730 & 1290 & 2355 & 2456 & 4780 & 9744 \\
\hline 4 & 689 & 1290 & 2601 & 1456 & 4799 & 7850 \\
\hline 5 & 800 & 1560 & 2546 & 3301 & 3450 & 8900 \\
\hline 6 & 823 & 1235 & 2284 & 2340 & 5320 & 9100 \\
\hline 7 & 760 & 1345 & 2300 & 2077 & 4590 & 9230 \\
\hline 8 & 900 & 1200 & 2456 & 2999 & 5377 & 8700 \\
\hline
\end{tabular}




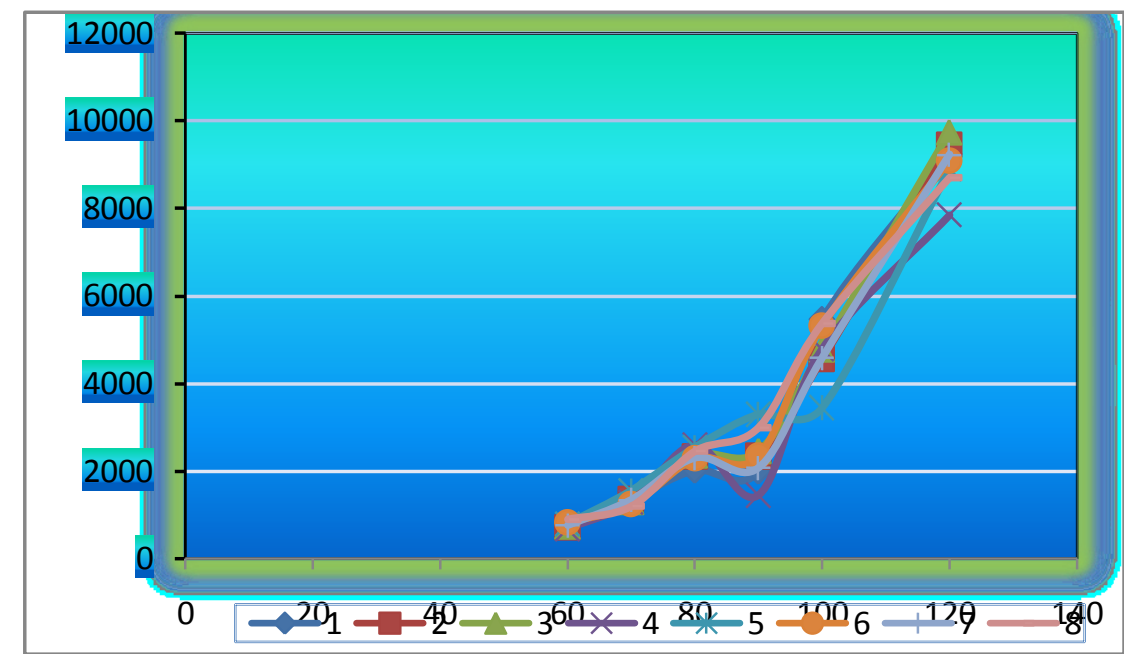

Fig (2) :Measured dose values of $8 \mathrm{x}$-ray Machine versus $\mathrm{kVp}$ for $40 \mathrm{mAs}$ at distance of $100 \mathrm{~cm}$

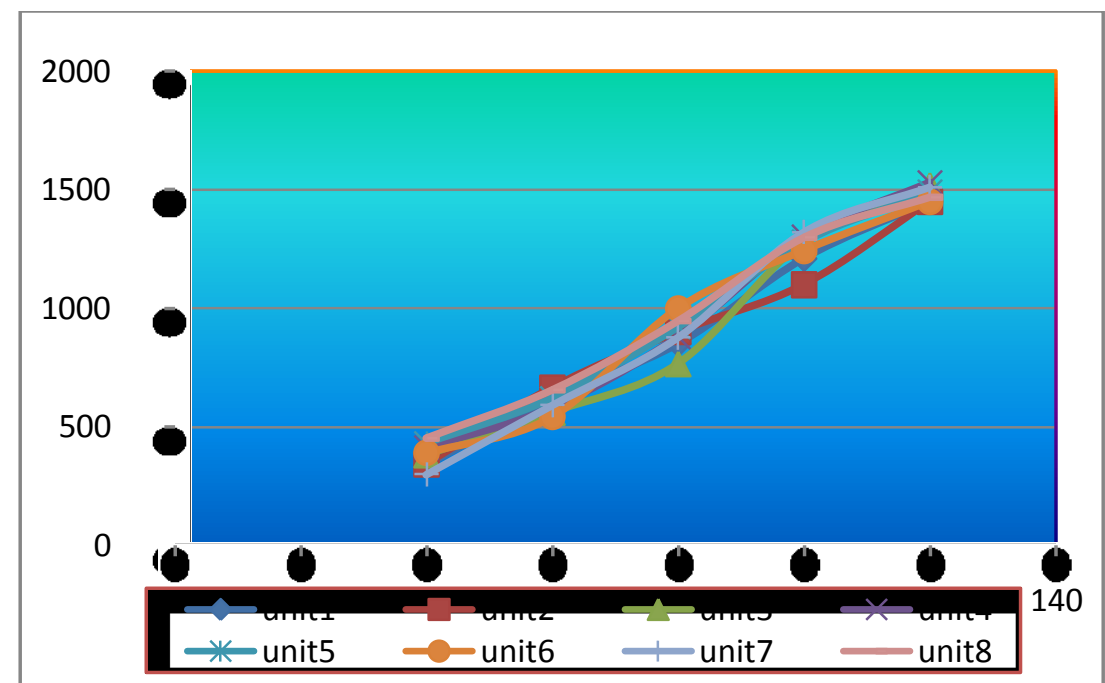

Fig (3) : Measured dose values of 8 x-ray Machine versus $\mathrm{kVp}$ for $40 \mathrm{mAs}$ at distance of $60 \mathrm{~cm}$

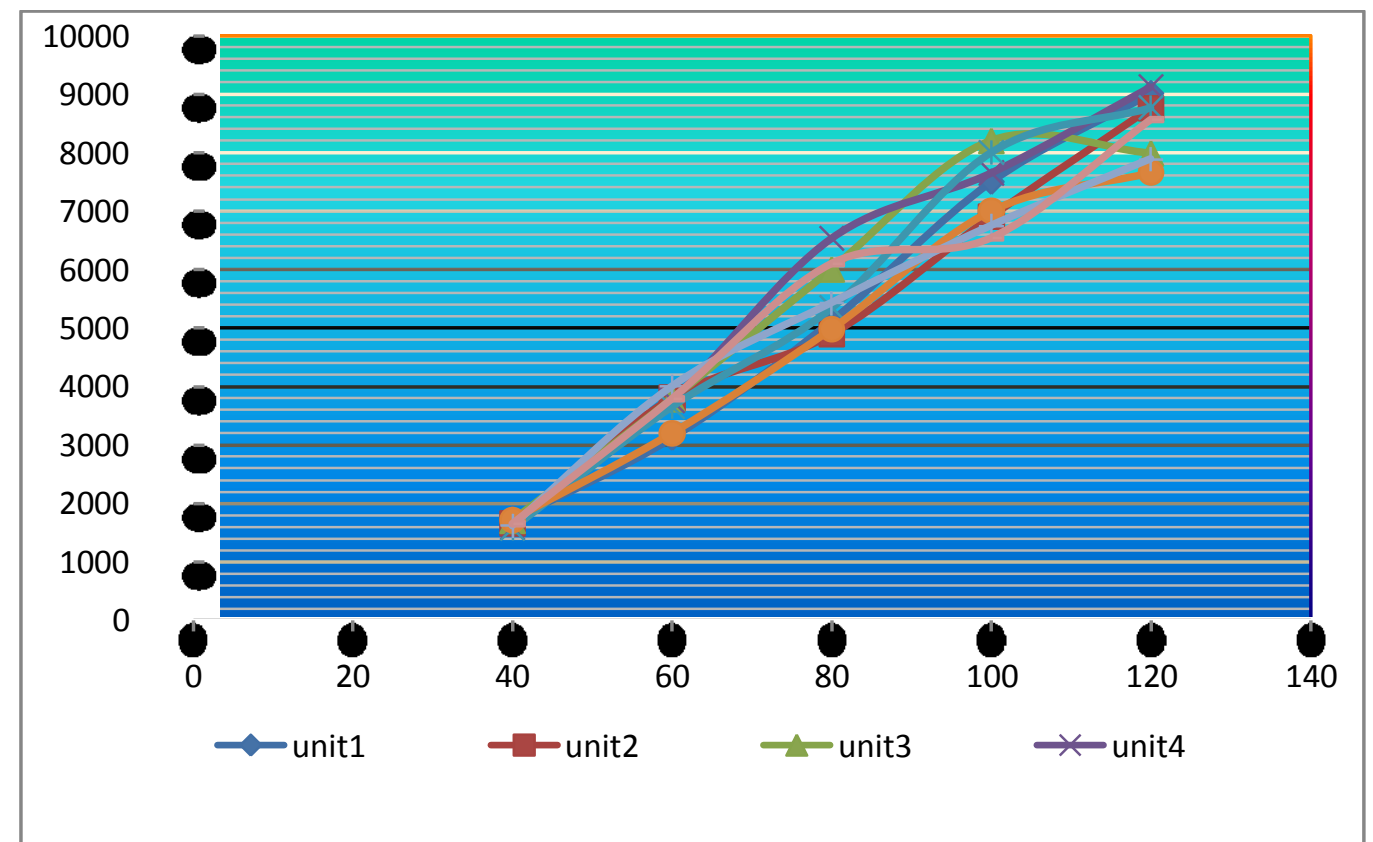

Fig ( 4) : Measured dose values of $8 \mathrm{x}$-ray Machine versus $\mathrm{kVp}$ for $60 \mathrm{mAs}$ at distance of $60 \mathrm{~cm}$ 


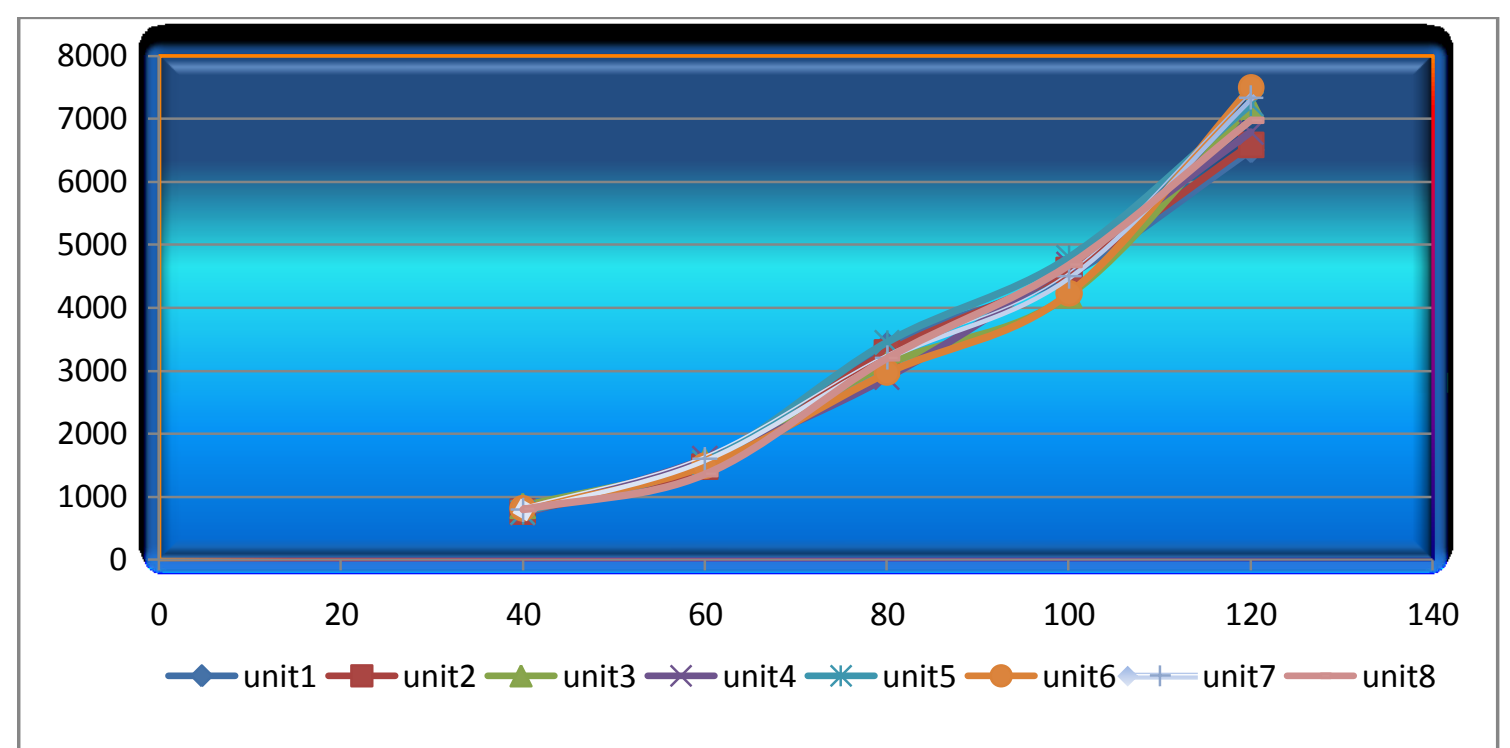

\section{Fig ( 5) : Measured dose values of $8 \mathrm{x}$-ray Machine versus $\mathrm{kVp}$ for $60 \mathrm{mAs}$ at distance of $100 \mathrm{~cm}$}

- $\quad$ For dose optimization, all exposures should be kept at the minimum dose level in according to the ALARA principle (ALARA-as low as reasonably achievable). The aim of the optimization is not to download the risks of irradiation to zero. It is to reduce them to an acceptable level. This can be possible only by examining all parameters that affect the X-ray, by investigating the relationship between dose and these parameters, on the basis of this relationship, by performing the necessary regulation

It is known that dose is more sensitive to the $\mathrm{kVp}$ changes than mAs changes. Exposure errors can occur if the actual $\mathrm{kVp}$ generated by the $\mathrm{x}$-ray generator is different from the adjusting $\mathrm{kVp}$ value. Before dose measurements, $\mathrm{kVp}$ accuracy testing was performed correctly and it was seen that the $\mathrm{kVp}$ during exposure was the close within the acceptable deviation to the selected $\mathrm{kVp}$ value.

All dose measurements were performed at different distance of $100 \mathrm{~cm}$ and $60 \mathrm{~cm}$. With this application, the distance effects on dose were investigated and it was used for dose modeling because of the inverse-square effect. For dose measurements, two different measurement procedures were used. In the first procedure, mAs value was kept constant and $\mathrm{kVp}$ values were changed to investigate the dose variation with $\mathrm{kVp}$. In the second procedure, $\mathrm{kVp}$ value was kept constant and $\mathrm{mAs}$ values were changed to investigate the dose variation with $\mathrm{mAs}$. Thus, the effects of $\mathrm{kVp}$ and $\mathrm{mAs}$ were examined separately.

Because the x-ray units were selected in according to the criteria's mentioned above, the measured dose values didn't show wide distribution for each measurement setup in all $8 \mathrm{x}$-ray Machines. In this condition, the mean of the dose values of $8 \mathrm{x}$-ray machines for each measurement setup was used to show the tube output variations with $\mathrm{kVp}$.

Risk factors for patients are different from risk factors for the general population and workers, due to several reasons. The risk estimates derived by ICRP (NRPB 1999) refer to a general population, considering both sex, a typical age distribution and typical cancer mortality rates. However, the age distribution for the exposed population to diagnostic radiology procedures presents a tendency to older age groups. The predominance of old or ill persons in a population, theoretically, would reduce the risk of long-term effects, in comparison with a population of workers. On the other hand, considering a population of children, that risk would be larger. However, the ICRP (1991) and the ICRP (1996) do not consider these facts; as an example, the ICRP (1996), in its paragraph 22, states 'Despite the wide range of organ doses in radiology and the differences in age structure, the collective dose and the nominal detriment coefficients provide a reasonably good indicator of the detriment in a population exposed in diagnostic radiology and nuclear medicine'.

\section{Conclusions}

The study showed variations in technique, exposure factors, film-screen combinations, and radiation dose for the same type of examination, both within and between rooms, which strongly supports the idea that further optimization is possible. Eighty hospitals recorded lower ESD values below EC/IAEA recommended diagnostic reference levels (10 mGy), and $40 \%$ of the hospitals exceeded the UK national reference value (4 mGy). Radiographic practices in Ghana are not fully optimized and this, therefore, calls for robust implementation of an appropriate and realistic QAprogram, which currently is not in existence in all the facilities surveyed. The variations in the data obtained also demonstrate the importance of creating awareness for the radiographic staff about regular quality control testing of the equipment and standardization of protocols, 
the urgent need for intervention and appropriate corrective actions in order to improve and standardize practice, enhancing the quality of the radiographs, and avoiding unnecessary risks of increased radiation dose to patients and staff. Also these variations, which are assumed to be present in most of the X-ray departments operating in the country, point to the need for the introduction of a national protocol and QA system, and frequent dose audits. A continuing need for bringing radiological procedures in Egypt in line with the "IAEA Guidelines on Quality Criteria for Diagnostic Radiological Imagesis worth emphasizing, in order to protect patients and staff from unnecessary radiation dose.

This study shows that optimization of technical factors may lead to a substantial dosereduction. If the optimized parameters are applied to X-ray equipment during quality control tests, it is possible to determine how much good image quality will be obtained with this optimized parameters and how much dose will be measured when this qualified image is developed. The results show the importance of radiographic staff training about the recommended parameters that are applied to the x-ray units for a qualified quality control system. It is essential to provide relevant education and training to staff in the radiology departments. It can be sure that with such a study the questions on many professional staff's mind will be answered, and the dose and the image characteristics will be parameters that are controlled and managed.

\section{References}

[1]. Sikand M, Stinchcombe S, Liversley PJ. Study on the use of gonadal protection shield during paediatrics pelvic X-rays. Ann R CollSurg Engl.;85(6):422-25, 2003.

[2]. Doolan A, Brennan CP, Rainford LA, Healy J. Gonad protection for antero-posterior projection of the pelvis in diagnostic radiography in Dublin hospitals. Radiography. 2004;10(1):15-21.

[3]. Hart D, Hillier MC, Wall BF. Doses to patients from medical X-ray examinations in the UK: 2000 review. NRPB W-14. Chilton, UK: National Radiological Protection Board; 2002.

[4]. Hart D, Hillier MC, Wall BF. Doses to patients from radiological and fluoroscopic X-ray imaging procedures in the UK-2005 review. HPA-RPD-029. Chilton, UK: Health Protection Agency, Radiation Protection Division; 2007.

[5]. International Commission on Radiological Protection. Radiological protection and safety in medicine.ICRP Publication 73. Ann ICRP. 2007;26(2):1-31.

[6]. Moores BM. Radiation dose measurement and optimisation [correspondence].Br J Radiol. 2005;78(933):866-68

[7]. Wall BF. Response to "Radiation dose measurement".Br J Radiol. 2006;79(940):356-58.

[8]. European Commission. Radiation Protection 109: Guidance on diagnostic reference levels (DRLs) for medical exposures. Luxembourg: OOPEC; 1999.

[9]. International Atomic Energy Agency (IAEA).Optimisation of the radiological protection of patients undergoing radiography, fluoroscopy and computed tomography. Report of a coordinated research project in Africa, Asia and Eastern Europe.IAEATECDOC-1423. Vienna, Austria: IAEA, 2004.

[10]. Škrk D, Zdešar U, Žontar D. Diagnostic reference levels for X-ray examinations in Slovenia.RadiolOncol. 2006;40(3):189-95.

[11]. European Commission. European guidelines on quality criteria for diagnostic radiographic images.EUR 16260 EN. Luxembourg: OOPEC; 1996.

[12]. American Association of Physicists in Medicine. Quality control in diagnostic radiology. AAPM Report 74. New York: Medical Physicists Publishing; 2002.

[13]. Institute of Physics and Engineering in Medicine. Recommended standards for the routine performance testing of diagnostic $\mathrm{x}$-ray imaging system. IPEM Report 91. York, UK: IPEM; 2005.

[14]. Dosimetry Working Party of the Institute of Physical Sciences in Medicine, National Radiological Protection Board.National protocol for patient dose measurements in diagnostic radiology. Chilton, UK: NRPB; 1992.

[15]. George J, Eatough JP, Mountford PJ, Koller CJ, Oxtoby J, Frain G. Patient dose optimization in plain radiography based on standard exposure factors. Br J Radiol. 2004;77(922):858-63.

[16]. Tsapaki V, Tsalafoutas A, Chinofoti I. Radiation doses to patients undergoing standard radiographic examinations: a comparison between two methods. Br J Radiol. 2007;80(950):107-12.

[17]. Ghana Statistical Services (GSS), Noguchi Memorial Institute for Medical Research (NMIMR), and ORC Macro. Ghana demographic and health survey 2003. Calverton, Maryland: GSS, NMIMR and ORC Macro; 2004. p. 11.Accessed from: http://www.measuredhs.com. Available by email from: reports@orcmacro.com

[18]. Johnston DA, Brennan PC. Reference dose levels for patients undergoing common diagnostic X-ray examinations in Irish hospitals. Br J Radiol. 2000;73(868):396-402.

[19]. Wall BF. Diagnostic reference levels - the way forward. Br J Radiol. 2001;74(885):785-88.

[20]. King S, Pitcher EM, Smail MA. Optimizing medical radiation exposures for uroradiological procedures, with special emphasis on paediatric imaging. Br. J Urology. 2002;89(6):510-16.

[21]. Minigh J. Paediatric radiation protection. Radiol Technol. 2005; 76 (5):365-75.

[22]. Ikamaise VC, Osuagwu CN, Bassey DE, et al. Assessment of chest radiographs for quality assurance and radiation protection. We st African J Radiol. 2000;7(1):18-25.

[23]. Bassey DE. Analysis of wasted x-ray films in the University of Calabar Teaching Hospital. West African J Radiol.1997;(4)1:28-32.

[24]. Schandorf C and Tetteh GK. Analysis of dose and dose distribution for patients undergoing selected X-ray diagnostic procedures in Ghana. RadiatProtDosim. 1998;76(4):249-56. 